\title{
Éditorial - II n'est jamais trop tard : styles de vie et vieillissement
}

De plus en plus, les gérontologues se préoccupent des questions de recherche suivantes: est-ce que les événements et expériences antérieurs jouent un rôle déterminant des résultats ultérieurs de la vie, surtout sur le statut-santé d'une personne ? Comment pouvons-nous encourager des comportements sains sur la période continue de la vie ? Les chercheurs empruntent les théories de plusieurs perspectives interdisciplinaires (par exemple, la théorie de la continuité, les approches développementales, la théorie du cheminement de la vie, la théorie du changement social et le modèle trans-théorique) afin de démontrer que le vieillissement sein n'est pas un événement immuable ; plutôt, il est le résultat de plusieurs tendances de vie étroitement reliées et en mutation constante. Ce modèle conceptuel se manifeste aussi dans les progrès réalisés en matières de styles de vie sains tels les comportements (par exemple, le conditionnement physique quotidien, une alimentation nutritive, le non-fumage et autres comportements à risque) associés à des résultats de santé positifs et de bien être. Par conséquent, les styles de vie sains sont considérés comme faisant partie d'un sous-ensemble de la pensée "style de vie. » L'Organisation mondiale de la santé offre la définition suivante: [a lifestyle is] a range of socially determined patterns of behaviour and interpretations of social situations, developed and used jointly by the group to cope with life (un style de vie est "une gamme de tendances comportementales sociales et de conjonctures sociales, réalisées et utilisées conjointement par le groupe afin de faire face à la vie ») (World Health Organization, 1986, p. 118). De cette perspective, les styles de vie sains et leurs comportements-santé particuliers, de même que le statut, les attentes sociales et les croyances-santé, forment une partie intégrante du cours de la vie d'un individu.

Les stratégies nationales, provinciales et régionales de santé ont adopté une perspective de santé de la population qui encourage, dans plusieurs politiques et programmes, un style de vie sain et la réduction des comportements à risque (par exemple, l'obésité). Toutefois, l'idée que les styles de vie sains sont le résultat $d$ 'un amas de chances et de choix de vie s'échelonnant sur la période de vie d'un personne peut avoir des connotations sérieuses, même si ces dernières sont involontaires, pour les individus se retrouvant à la cyme de la pyramide-âge. Il semblerait y avoir une supposition qui avance que la meilleure valeur ajoutée de nos efforts de promotions de santé se réalisera au sein des personnes plus jeunes, étant donné que ces dernières peuvent tirer profit de leurs actions sur une période prolongée. Par exemple, si une jeune personne arrête de fumer, il peut y avoir des avantages à long terme pour sa santé surtout en matière de réduction de risque de maladies; pour la société, les avantages reposent sur une réduction de réclamations auprès du système de santé. Toutefois, l'accentuation excessive sur les comportements-santé dès les débuts de la vie, peuvent dévaluer et même décourager les politiques et les actions de modification de style de vie au sein des personnes accédant aux âges avancés. La recherche considérable démontre que les avantages santé associés à un style de vie amélioré, s'accumulent même si les individus sont des personnes âgées. Malgré tout, il y a hésitation à exécuter des politiques et des programmes de promotion de telle façon à rencontrer les besoins d'une population âgée de plus en plus hétérogène. Sans aucun doute, il est difficile de renverser le stéréotype qui dépeint les personnes âgées comme étant plutôt inactives et moins prédisposées à effectuer des changements sains à leur style de vie ; ceci empêche la réalisation de ces buts au sein d'une société misée sur la jeunesse. Tournons notre attention au conditionnement physique - un comportement éprouvé, appuyant un style de vie sain. Nous utiliserons le terme exercice indifféremment du terme activité physique, quoiqu'il est reconnu que ces derniers détiennent des définitions et des fonctionnements différents dans la littérature.

Le premier article du présent numéro de la Revue canadienne du vieillissement intitulé "Do "Young-Old" Exercisers Feel Better Than Sedentary Persons? A Cohort Study in Switzerland » de Lalive, d'Epinay et Bickel, introduit des résultats pertinents à cette discussion. L'étude appui un lien causal entre les trajectoires sports/exercices lorsque mesurées entre la quarantaine (prés de l'âge 50) et les jeunes-âgées (de 65 à 74 ans), et les résultats du bien être à ces âges avancés. Les auteurs proposent quatre catégories de gens actifs: les sportifs constants (ceux entre la quarantaine et l'âge des jeunes-âgés qui pratiquent un conditionnement physique au moins une fois par semaine); les nouveaux sportifs (ceux qui étaient sédentaires à la quarantaine et qui deviennent actifs ); les anciens sportifs (ceux qui pratiquaient un conditionnement physique à la quarantaine mais qui ont cessé à l'âge des jeunes-âgés); et les sédentaires (ceux qui sont sédentaires et à la quarantaine et à l'âge de jeune-âgé). Les méthodes quantitatives de bien être comprennent l'auto évaluation de santé et la Wang self-assessed depression scale. Plus précisément, les 
auteurs démontrent que, tel qu'attendu, les habitués font preuve de niveaux avancés de bien être comparativement aux lâcheurs et aux sédentaires. La découverte que les nouveaux venus considèrent leur santé à un niveau rapprochant les habitués intrigue les chercheurs. Ce résultat suggère qu'un individu peut se rattraper, du moins du point de vue des avantages socio-psychologiques du conditionnement physique. Finalement, il convient de noter que presque 40 pour cent de l'échantillon de l'étude effectuait une migration entre une personne physiquement active à une personne non-active et vice versa durant cette même période.

Ces résultats sont compatibles à une littérature considérable de recherche qui avance des preuves irréfutables qu'un style de vie actif a des avantages positifs sur la santé physique et subjective au sein des personnes de tout âge, y compris les personnes âgées et les très âgés. Les résultats démontrent non seulement l'existence d'un lien causal entre les trajectoires du conditionnement physique au cours de la vie et le statut-santé, mais appuyent la notion que le comportement de conditionnement physique n'est pas un état stable tout au long de la vie d'une personne. En fait, les tendances de conditionnement physique semblent être plutôt variables. Ceci est semblable à d'autres recherches qui démontrent que le conditionnement physique est influencé par l'association réciproque de ce dernier et les changements en statut-santé (et autres caractéristiques) lors du vieillissement. Par exemple, un style de vie actif peut retarder de prime abord la maladie et la maladie ou une blessure peut exercer un effet dissuasif contre le conditionnement physique. D'ailleurs, la recherche des auteurs démontre que les individus qui commencent un conditionnement physique entre 50 ans et 60 à 70 ans, prennent avantage des bénéfices naturels d'un style de vue sain.

La question est la suivante : est-ce que nos efforts de promotions de santé aident adéquatement les individus à initier, soutenir et ranimer, si c'est le cas, un style de vie sain ? Du côté positif, le pourcentage de la population qui se prête à des niveaux de conditionnement physique sédentaires ou peu fréquents a baissé d'une manière importante depuis les années 70 depuis nos jours (Wister, 2003). Dans cette recherche, le conditionnement physique sédentaire ou peu fréquent se défini arbitrairement comme étant d'une fréquence de moins de trois intervalles d'exercice par semaine et étant d'une durée de 15 minutes ou plus. Ce niveau est considéré comme étant inférieur au niveau minimum recommandé et par conséquent, démontre un comportement de style de vie malsain. De plus, depuis les dernières 25 années et allant à l'encontre des croyances populaires, cette baisse des niveaux malsains de conditionnement physique s'est manifestée à peu près au même rythme pour les personnes âgées et les autres adultes. Du côté négatif, la proportion de la population suivant des directives minimales de pratique de style de vie sain de base est loin d'être suffisante lorsque examinée de la perspective population-santé. Pour ce qui est des niveaux de conditionnement physique, l'on estime que 31 pour cent des femmes âgées et 41 pour cent des Canadiennes et des Canadiens âgés de 65 ans et plus, sont modérés ou plus en matière de dépense d'énergie attribuable à l'activité physique de temps libres ; ce niveau est souvent associé à une variété d'avantages santé (Health Reports, 1999). De plus, les niveaux de conditionnement physique ne sont pas plus élevés pour ceux appartenant à la cohorte des enfants du baby-boom (ces derniers commenceront à franchir le 65 ans par l'an 2011) ni pour la population entière.

Qu'est-ce qui nous empêche de réaliser et de soutenir des styles de vie actifs tout au long du cycle de vie? Nous détenons une vaste littérature qui se penche sur les mécanismes causals sous-jacents les tendances d'activités physiques (voir par exemple, le survol de la littérature de Sherwood et Jeffery, 2000). Il va sans dire que l'activité physique est un processus complexe et dynamique, influencée par plusieurs caractéristiques individuelles, sociales et environnementales, y compris les obstacles (par exemple, l'auto-efficacité inférieure) et les facilitateurs (par exemple, l'appui familial positif) aux processus d'activité physique. De cette recherche nous pouvons conclure qu'en toute probabilité, une approche multi-disciplinaire de la vie active, acceptant l'hétérogénéité des gens de tout âge, produira les améliorations les plus élevées en matière santé. Cette approche occasionnerait des politiques et des programmes qui dépassent les contextes liés aux individus, aux groupes sociaux et à la société.

Ceci demande un épanouissement et une mise en valeur des programmes clés de conditionnement physique qui rencontrent les besoins, les ressources et la prédisposition au changement des individus, et ce à des moments divers de leur période de vie et comprenant des conditions de santé et des systèmes d'appui variés et des classes sociales et ethnies différentes. Pour ce qui est des personnes âgées, les programmes structurés de conditionnement physique devraient prévoir un certain pourcentage de décrochage, de même que des améliorations aux méthodes de recrutement et d'entretien des participants qui manifestent une gamme de changements ou de symptômes de maladies relatifs à l'âge. Ces programmes d'activités doivent être agréables, sembler attrayants aux individus manifestant des goûts et des préférences variées et comprendre les composantes sociales afin de consolider l'engagement et la participation continue. De plus, compte tenu de leur rôle influant dans 
la société, les professionnels de la santé, surtout les omnipraticiens et les médecins de famille, doivent participer davantage à l'appui de l'adoption ou de la continuité d'un style de vue sain. Ils peuvent aider les gens à comprendre les directives de pratiques d'excellence appartenant à l'exercice, la nutrition et les interactions de médicaments.

D'une façon générale, il est peu douteux que les attitudes sociales doivent changer. Les efforts visant à augmenter d'une façon importante les niveaux d'activités physiques des personnes de tout âge par l'entremise de campagnes nationales, doivent reconnaître le besoin d'intégrer le conditionnement physique au style de vie ; toutefois, ce comportement doit être soutenu par le jeune adulte, celui dans sa quarantaine et la personne âgée. Au niveau de la politique, les gouvernements doivent démontrer un engagement considérable envers une société saine et active ceci est prioritaire. La construction et mise en œuvre de pistes cyclables et de pistes pédestres sécuritaires, d'espaces verts et de parcs sont des exemples concrets d'une infrastructure manœuvrable qui encourage des styles de vie actifs. En conclusion, il est de mise de disséminer un message positif et puissant qui renseigne et éduque les gens qu'il n'est jamais trop tard pour adopter ou pour renouveler un style de vie sain.

Andrew V. Wister

Programmes de gérontologie

Université Simon Fraser

\section{Références}

Health Reports (1999). Personal health practices: Smoking, drinking, physical activity and weight. Health Reports, 11(3), 83-90.

Sherwood, N. et Jeffery, R. (2000). The behavioural determinants of exercise: Implications for physical activity interventions. Annual Review of Nutrition, 20, 21-44.

Wister, A.V. (2003). Baby boomer health dynamics: How are we aging? Manuscrit non-publié.

World Health Organization. (1986). Lifestyles and health. Social Science and Medicine, 22, 117-124. 